\title{
Extracerebral metastases of glioblastoma have a different vasculature than primary tumour. A case report of glioblastoma extracranial metastases
}

\author{
Dorota Snopkowska-Wiaderna ${ }^{1}$, Krzysztof W. Zieliński', Maciej Radek², Wielisław Papierz ${ }^{3}$ \\ 1Department of Pathomorphology and Clinical Cytopathology, Medical University of Łodz, Poland, ${ }^{2}$ Clinics of Neurosurgery and Peripheral \\ Nerve Surgery, Medical University of Łodz, Poland, ${ }^{3}$ Department of Pathomorphology, Medical University of Łodz, Poland
}

\begin{abstract}
The paper presents a case report of a 38-year-old female suffering from metastatic glioblastoma in the jugular lymph node that developed 9 months after craniotomy and tumorectomy in the left temporal region of the brain. The histological evaluation of metastatic tumour reveals lower density of vasculature as well as less significant pathologic changes in blood vessels morphology in comparison to primary tumour. Moreover, in this report we present cytological characteristics of the material obtained by fine needle aspiration of the metastatic mass.
\end{abstract}

Key words: glioblastoma, extracranial metastases, vasculature, fine needle biopsy.

\section{Introduction}

Although glioblastoma is the most aggressive glioma, it rarely gives metastases located outside the central nervous system. In the literature only as many as several hundred reports of extracranial metastases of glioblastoma cases can be found. The most often localizations of metastases are: lungs, lymph nodes of the neck [1], bones [8], liver, and spleen, stomach and colon. Extracranial metastases typically occur in younger patients, who had tumorectomy and radio- and chemotherapy treatment $[1,5,6]$. The cause of the rarity of extracranial metastases in such low-differentiated tumour is still unknown [7], therefore the analysis of each case seems to be justified.

\section{Case report}

A 38-year-old woman was admitted to hospital due to severe headaches, memory and speech disorders, with the history of an epileptic seizure. Her MRI showed the cystic-solid tumour located in the left temporal lobe of the brain with infiltration of the island accompanied by brain oedema. After being referred to the surgery, left frontal-temporal craniotomy was performed and the tumour was excised. The histopathological examination of the tumour showed a typical picture of glioblastoma, WHO grade IV. The patient was treated with radiotherapy (total dose: 6000 cGy) and five cycles of chemotherapy based on Temodal (140 mg per day). 
Nine months after the surgery the patient came with hard and painful tumour of a diameter of $6 \mathrm{~cm}$, knit with the ground and located on the left side of the neck, which has been removed. One month later in the operated area of the neck, a recurrence tumour $(13 \times 10 \mathrm{~cm})$ was found.

An angio-CT image showed a nodular packet of lymph nodes (dimensions of $6 \times 6 \times 4.5 \mathrm{~cm}$ ), infiltrating nearby tissue, located in the left part of the side of the neck. Its total resection was performed. Histopathological examination of both tumours preceded by a fine needle biopsy of the tumour of the neck showed malignant neoplasm that could be a spread of extracranial glioblastoma. The patient died 19 months after the initial cranial glioblastoma surgery.

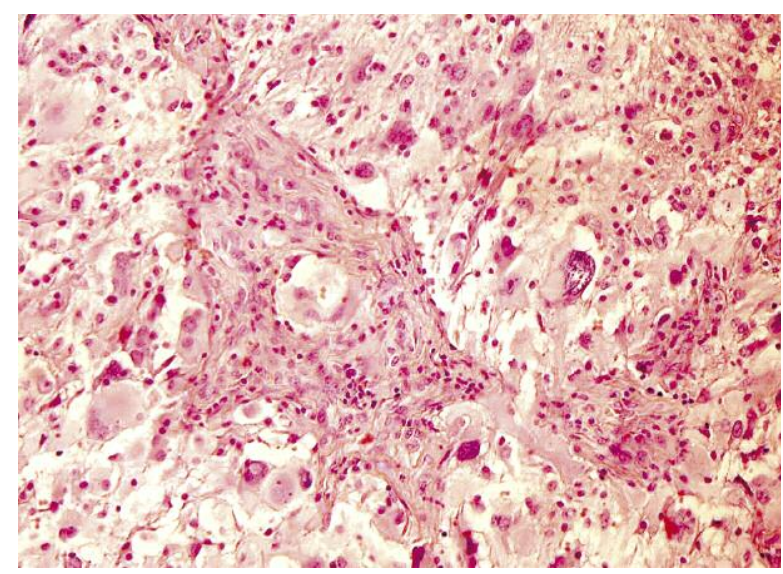

Fig. 1. Cerebral tumour. Note the pathology and proliferation of blood vessel's wall cells. Haematoxylin eosin. Magn. $\times 400$.

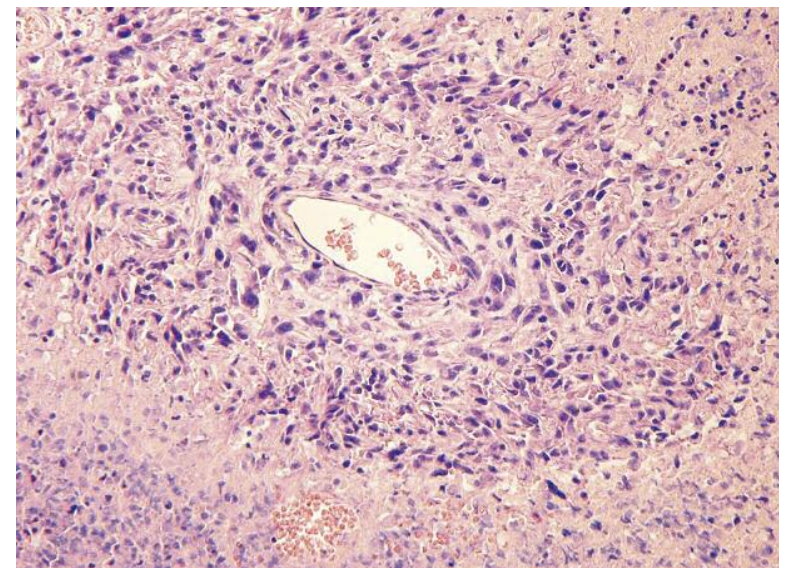

Fig. 2. Metastatic tumour. Note the difference of the structure of the vessel's wall, as compared to Fig. 1. Haematoxylin eosin. Magn. $\times 200$.

\section{Pathologic findings}

Microscopic examination of the primary tumour, which was located in the left temporal lobe, showed a malignant neoplasm - glioblastoma, WHO grade IV. The tumour had typical diagnostic morphological features, recommending identification of this type of neoplasm (1): high grade cytological atypia, numerous pathological mitoses, presence of many multinuclear, giant and bizarre cells, areas of coagulative necrosis, haemorrhages and numerous thick-wall vessels with the proliferation of endothelial cells. The immunohistochemical profile was also typical of multiform glioblastoma: GFAP (+), S-100 (+), vimentin (+), EMA $(-)$, cytokeratins $(-)$. In small parts of the tissue, some areas of oligodendroglioma were found, confirmed by olig2 immunohistochemical reaction.

In the post-operative material from the tumours located on the left side of the neck, histopathological similarity to the microscopical image of the temporal lesion was found. In addition there were found: areas of spindle cells with hyperchromatic atypical nuclei, gemistocytic component, argentophilic fibres (Gomori's silver impregnation), which resemble the structure of sarcoma and are likely to be gliosarcoma. In comparison with the brain tumour (Fig. 1), different morphology of blood vessels in metastatic tumour was noted. Vessels in the metastatic tumour were much less numerous and narrower (similar in appearance to capillaries), endothelial cells were flat, less polymorphic and without stratification (Fig. 2).

The cytological image of neck tumour specimens is consistent with fusocellular sarcoma (Fig. 3). Groups of fusiform, elongated cells with hyperchromatic nuclei, sometimes with coarsely granular or clumped chromatin, prevailed in the microscopic image. Apart from that, single, much bigger, polymorphic cells with large, homogeneously hyperchromatic nuclei (sometimes with a vacuole) and with abundant acidophilic cytoplasm were found. The background of cytological image was fibrillar. In comparison with descriptions of the cytological image of squash specimens of glioblastoma [7], there were neither many vascular bands nor close relationship between tumour cells and them.

In order to determine the origin of the neck tumour, the following immunohistochemical tests were performed: GFAP (+), CK (-), VIM (-), CD34 (-), CD68 (-), thyroglobulin (-), TTF-1 (-) and melan-A (-).

In comparison with the brain tumour, the GFAP immunohistochemical test in metastatic tumour cells was 
less intense and present in only about $80 \%$ of the cells (Fig. 4).

The above summary of the histological characteristics and immunohistochemistry of the neck tumour suggests the presence of extracranial glioblastoma.

\section{Discussion}

Despite a relatively high incidence (annually 3-4 cases per 100000 population) [5], and high grade atypia, glioblastoma rarely gives extracranial metastases. Other cancers of the brain, in which extracranial metastases were described, are medulloblastoma, ependymoma and oligodendroglioma [10]. Those described cases of extracranial glioblastoma usually involved people who underwent neurosurgical treatment comprised of craniotomy, tumorectomy and even stereotactic biopsy that damaged brain vessels, meninges and skull bones. There was also a hypothesis, which connects the metastatic process with chemo- and radiotherapy that the patient was treated with. In the opinion of its authors, chemo- and radiotherapy have an impact on the nature and function of the neoplastic glioma cell [6]. In the presented case, the patient was treated with Temodal (140 mg a day) and radiotherapy in the total dosage of $6000 \mathrm{cGy}$.

The discussed causes of rare occurrence of extracranial metastases are rapid progress of the disease (usual survival time is too short for the metastases to occur), immune response to tumour cells located outside the central nervous system, absence of so far unknown factors that enable growth outside the central nervous system, obstacles that impede cells infiltration outside the central nervous system [7], including the existence of the blood-brain barrier. It is presumed that there is a specific composition of proteins (possibly secreted by astrocytes [7]) in the basement membrane of brain vessels that impedes the cells infiltration to vessel lumen [1]. The ways of glioblastoma spreading outside the central nervous system have not been determined yet [9]. The fact that the majority of the metastases of this neoplasm affect lymph nodes suggests the participation of cerebral meninges lymph vessels in this process $[4,9]$. This hypothesis is probable in surgical patients, but it is hard to accept it in non-operated patients with lymph nodes metastases [9]. There are many studies which showed glioblastoma dispersal through blood vessels, which was confirmed in immunohistochemical assays with CD34 antibody [2]. Intracerebral glioblastoma have a unique and characteristic

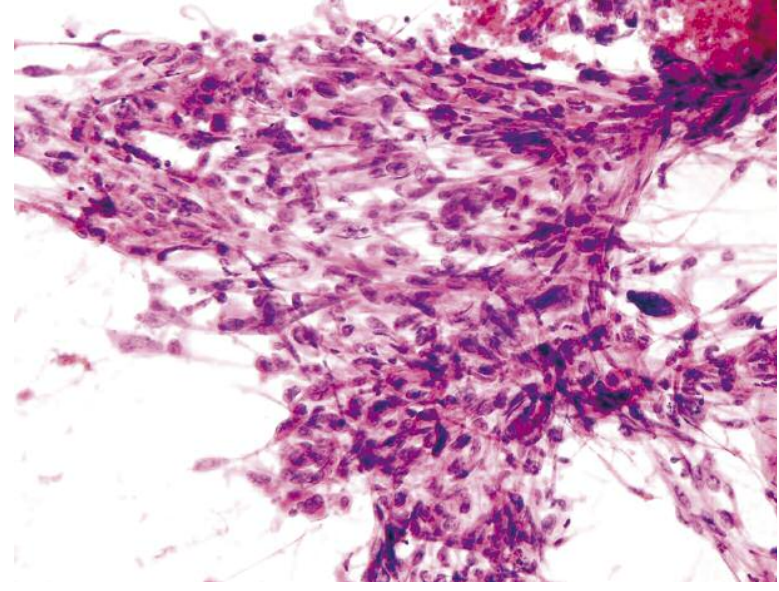

Fig. 3. Microscopic image of cytological smear of metastatic tumour of the neck. Haematoxylin eosin, fine needle biopsy. Magn. $\times 400$

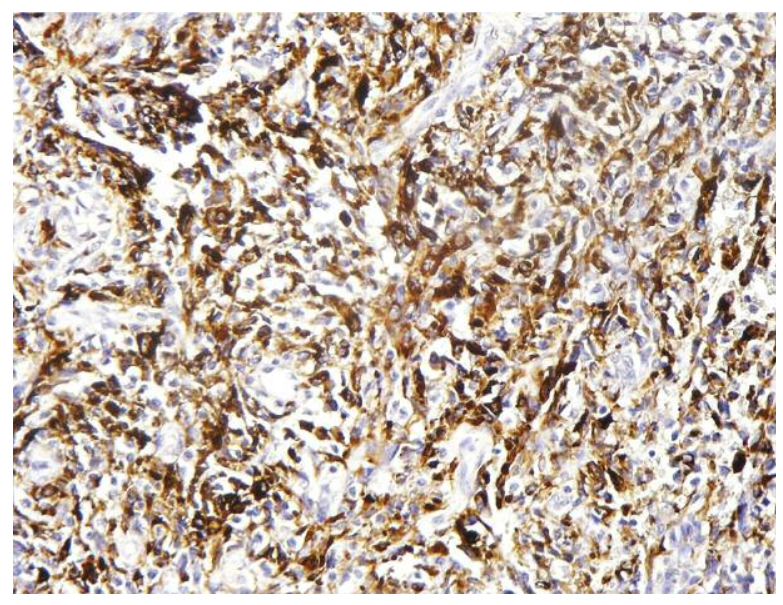

Fig. 4. GFAP positive cells of metastatic tumour. Magn. $\times 200$

vascular network [1,3]. Neoplastic vessels are thickwalled with several layers of high endothelial cells. They form irregular tangle glomerular structures that are linked and arranged chaotically. As a result, there are circulatory disturbances, local hypoxia with low $\mathrm{pH}$ in the tumour [11] and increased blood vessels' wall permeability. Vessel proliferation is stimulated by the tumour itself, which produces growth factors and cytokines. The most known is vascular endothelial growth factor (VEGF), concentration of which is fiftyfold higher in glioblastoma, in comparison to healthy central nervous tissue [4].

In the discussed case, vessels' structural changes were not as intense in metastatic tumour as in the primary lesion. Apart from the difference in the vessel net- 
work, metastatic tumour had also an admixture of gliosarcoma and gemistocytic component.

In conclusion, we would like to draw the attention to an underestimated possibility of finding metastases of glioblastoma in extracranial organs. In the described case there is an essential difference in the structure of the tumours depending on their localisation, especially concerning the morphology of blood vessels, which suggests a possible impact of brain tissue environment on the conformation of these vessels.

\section{References}

1. El-Gindi S, Salama M, El-Henawy M, Farag S. Metastases of glioblastoma multiforme to cervical lymph nodes: report of two cases. J Neurosurg 1973; 38: 631-634.

2. Frank S, Kuhn SA, Brodhun M, Mueller U, Romeike B, Kosmehl H. Metastatic glioblastoma cells use common pathways via blood and lymphatic vessels. Neurol Neurochir Pol 2009; 43: 183-190.

3. Frontczak-Baniewicz M, Czajkowska D, Andrychowski J, Walski M. The immature endothelial cell in human glioma. Ultrastructural features of blood capillary vessels. Folia Neuropathol 2008; 46: 49-56.

4. Jain RK, di Tomaso E, Duda DG, Loeffler JS, Sorensen AG, Batchelor TT. Angiogenesis in brain tumors. Nat Rev Neurosci 2007; 8: 610-622.

5. Kleihues P, Burger PC, Aldape KD, Brat DJ, Biernat W, Bigner DD. Glioblastoma. In: Louis DN, Ohgaki H, Wiestler OD, Cavenee WK (eds.). World Health Organization Classification of Tumours of the Central Nervous System. IARC, Lyon 2007.

6. Mirimanoff RO, Gorlia T, Mason W, Van den Bent MJ, Kortmann RD, Fisher B, Reni M, Brandes AA, Curschmann J, Villa S, Cairncross G, Allgeier A, Lacombe D, Stupp R. Radiotherapy and temozolomide for newly diagnosed glioblastoma: recursive partitioning analysis of the EORTC 26981/22981-NCIC CE3 phase III randomized trial. J Clin Oncol 2006; 24: 2563-2569.

7. Mourad PD, Farrell L, Stamps LD, Chicoine MR, Silbergeld DL. Why are systemic glioblastoma metastases rare? Systemic and cerebral growth of mouse glioblastoma. Surg Neurol 2005; 63: 511-519.

8. Utsuki S, Tanaka S, Oka H, Iwamoto K, Sagiuchi T, Fujii K. Glioblastoma multiforme metastasis to the axis. Case report. J Neurosurg 2005; 102: 540-542.

9. Wallace CJ, Forsyth PA, Edwards DR. Lymph node metastases from glioblastoma multiforme. AJNR 1996; 17: 1929-1931.

10. Yokosuka K, Ishii R, Suzuki Y, Hirano K, Ishii N, Sekihara Y, Hamazaki S, Nishimura H. Extraneuronal metastasis of high grade glioma without simultaneous central nervous system recurrence. Neurol Med Chir (Tokyo) 2007; 47: 273-277.

11. Yokoyama H, Ono H, Mori K, Kishikawa M, Kihara M. Extracranial metastasis of glioblastoma with sarcomatous component. Surg Neurol 1985; 24: 641-645. 